
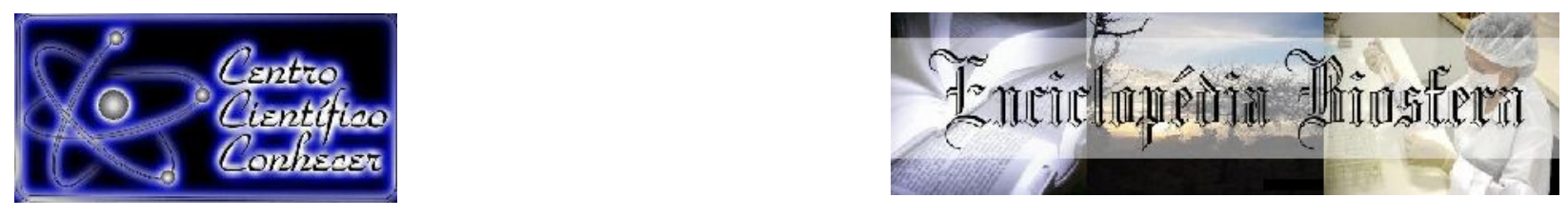

\title{
PERSISTÊNCIA DE DENTES DECÍDUOS E PRESENÇA DE DENTES INCLUSOS E CISTO DENTÍGERO EM UM CÃO SHIH-TZU ADULTO
}

Natália Saraiva Rodrigues ${ }^{1}$, Kelli Cristina Graciano ${ }^{2}$, Kimberlly Karoline Corrêa da Cruz $^{2}$, Rogério Ribas Lange ${ }^{3}$, José Ricardo Pachaly ${ }^{4}$

1. Mestranda em Ciência Animal, Universidade Paranaense - UNIPAR, bolsista PIT/UNIPAR (nataliasrodrigues123@hotmail.com), Umuarama, PR, Brasil.

2. Médicas Veterinárias Residentes em Odontologia Veterinária, Universidade

Federal do Paraná - UFPR, Curitiba, PR, Brasil.

3. Professor Doutor, Programa de Pós Graduação em Ciências Veterinárias, Universidade Federal do Paraná - UFPR, Curitiba, PR, Brasil.

5. Professor Pós-Doutor, Programa de Pós Graduação em Ciência Animal, Universidade Paranaense - UNIPAR, Umuarama, PR, Brasil.

Recebido em: 06/04/2019 - Aprovado em: 10/06/2019 - Publicado em: 30/06/2019 DOI: 10.18677/EnciBio_2019A100

\begin{abstract}
RESUMO
A persistência de dentes decíduos é uma condição comum e acomete principalmente cães de raças pequenas e miniaturas. Pode levar a diversas alterações orais, sendo indicada a extração desses dentes decíduos persistentes. Dentes inclusos são aqueles cuja erupção não ocorreu, e devem ser extraídos para evitar complicações como a formação de cistos odontogênicos. Os cães braquicéfalos são os mais suscetíveis, e o cisto dentígero é o cisto odontogênico mais comum em cães. Mesmo sendo benigno, pode alcançar tamanhos significativos, levando a diversas alterações locais. Este artigo relata um de caso sobre persistência de três dentes decíduos e presença de dois dentes inclusos, sendo um deles associado a um cisto dentígero, em um cão Shuh-Tzu adulto. Discute-se diagnóstico, tratamento cirúrgico e evolução do caso.
\end{abstract}

PALAVRAS-CHAVE: exodontia, extração dental, odontologia, odontologia veterinária.

\section{PERSISTENCE OF DECIDUOS TEETH AND PRESENCE OF IMPACTED TEETH AND A DENTIGEROUS CYST IN A SHIH-TZU ADULT DOG}

ABSTRACT

Persistence of deciduous teeth is a common condition and mainly affects small breeds and toy dogs. It can lead to several oral changes, being indicated the extraction of these persistent deciduous teeth. Impacted teeth are those which not erupted, and should be extracted to avoid complications such as odontogenic cysts. Brachycephalic dogs are the most susceptible, and dentigerous cyst is the most common odontogenic cyst in dogs. Although benign, it can reach significant sizes, leading to several local changes. This article reports a case of persistence of three ENCICLOPÉDIA BIOSFERA, Centro Científico Conhecer - Goiânia, v.16 n.29; p. 1603 
deciduous teeth and presence of two impacted teeth, being one of them associated to a dentigerous cyst, in an adult Shih-Tzu dog. Diagnosis, surgical treatment and case evolution are discussed.

KEYWORDS: exodontics, tooth extraction, veterinary dentistry.

\section{INTRODUÇÃO}

O conhecimento anatômico, em especial sobre a dentição normal e o período de substituição dentária, permite identificar muitos casos de alterações ou enfermidades odonto-estomatológicas (NIEMIEC, 2008; KRESSIN, 2009). Nessa abordagem, utilizam-se principalmente dois sistemas de identificação dental - o Sistema de Identificação Anatômico do Dente e o Sistema Triadan modificado (KRESSIN, 2009; FULTON et al., 2014).

No primeiro, as letras indicam a que dente se refere a anotação, sendo que o número superior equivale à quantidade de dentes maxilares de uma das hemiarcadas (direita ou esquerda), e o número inferior corresponde aos dentes mandibulares. Assim, para se obter o total de dentes das hemiarcadas deve-se multiplicar a soma por dois, pois estas são simétricas (KRESSIN, 2009; FULTON et al., 2014). Já no sistema Triadan modificado, cada dente recebe um número de três dígitos, no qual o primeiro dígito indica a qual quadrante pertence - 100 para a maxila direita, 200 para maxila esquerda, 300 para mandíbula esquerda e 400 para mandíbula direita. Em se tratando de dentição decídua, acrescenta-se 400 a cada quadrante, sendo que os dois últimos dígitos mostram a posição anatômica - por exemplo, todos os caninos são numerados como 04 (KRESSIN, 2009; FULTON et al., 2014).

A erupção dos dentes decíduos nos cães começa a partir da terceira semana de vida e se completa aos dois meses de idade, período em que geralmente ocorre o primeiro exame médico do filhote (DOMNICK, 2014; FULTON et al., 2014; SANTOS et al., 2014). Os cães possuem 28 dentes decíduos, sendo 12 incisivos, quatro caninos e 12 pré-molares, não existindo primeiros pré-molares nem molares decíduos (KRESSIN, 2009; FULTON et al., 2014, SANTOS et al., 2014).

Entre dois e seis meses de idade observa-se uma dentição mista, composta por dentes decíduos e permanentes, sendo possível diferenciá-los pelo fato dos dentes decíduos serem menores, mais afilados e pontiagudos (FULTON et al., 2014; SANTOS et al., 2014). Aos seis meses de idade completa-se a erupção de todos os 42 dentes permanentes, sendo 12 incisivos, quatro caninos, 16 pré-molares, quatro molares maxilares e seis molares mandibulares (KRESSIN, 2009; FULTON et al., 2014; SANTOS et al., 2014).

À medida que ocorre a erupção do dente permanente, a reabsorção do dente decíduo correlato é estimulada, e quando o processo de reabsorção é lento ou não ocorre, observa-se persistência de determinados dentes decíduos (FULTON et al., 2014; SANTOS et al., 2014), que é comum especialmente nas raças pequenas e miniaturas (NIEMIEC, 2008; SANTOS et al., 2014). Os fatores predisponentes incluem causas genéticas, má oclusão, deficiências nutricionais, alterações metabólicas, distúrbios endócrinos, presença de polpa necrótica com consequente reabsorção lenta dos dentes decíduos, e posição ectópica do botão germinativo de dente permanente (NIEMIEC, 2008; FULTON et al., 2014; SANTOS et al., 2014).

A persistência de dentes decíduos pode ocasionar má oclusão, mordida cruzada, predisposição a doenças periodontais, dor, dificuldade na alimentação, acúmulo de cálculo dental, apinhamento dental e alterações gengivais. Assim, ENCICLOPÉDIA BIOSFERA, Centro Científico Conhecer - Goiânia, v.16 n.29; p. 1604 
recomenda-se realizar exame oral cuidadoso em filhotes com até seis meses de idade, para que essa condição seja identificada a tempo de reduzir suas eventuais consequências (NIEMIEC, 2008; FULTON et al., 2014; SANTOS et al., 2014). O diagnóstico da persistência de dentes decíduos é baseado no exame físico da cavidade oral, sendo ainda úteis as radiografias intra-orais (SANTOS et al., 2014).

O tratamento da persistência de dentes decíduos consiste na exodontia, tomando-se cuidado para evitar danos ao periodonto e aos dentes permanentes (NIEMIEC, 2008; FULTON et al., 2014, MOORE; NIEMIEC, 2014; SANTOS et al., 2014). Antes da extração dental é primordial realizar radiografias intra-orais, que permitem determinar o tamanho e formato de cada dente e verificar a presença de reabsorção ou anquilose. Uma das principais complicações é a fratura de raiz, que deve ser totalmente removida para evitar a ocorrência de inflamação e abscessos (FULTON et al., 2014; SANTOS et al., 2014; NIEMIEC, 2015; PERRY, 2017).

Quando houver ausência de dentes em um cão é essencial a realização de radiografias para investigar a causa etiológica, que pode estar ligada a agenesia dentária, extração prévia, fratura dental com retenção de raiz e dente incluso ou impactado (LEITE et al., 2011; KIM et al., 2013; DOMNICK, 2014; FULTON et al., 2014; HONZELKA et al., 2014; NIEMIEC, 2015; BABBITT et al., 2016; PERRY, 2017).

Dentes inclusos são aqueles cuja erupção não ocorreu, geralmente devido a falha nas forças de erupção e barreiras físicas, que podem levar inclusive a mau posicionamento dental do incluso (KRESSIN, 2009; EDSTROM et al., 2013; CARLE; SHOPE, 2014; DOMNICK, 2014; FULTON et al., 2014; HONZELKA et al., 2014; NIEMIEC, 2015; BABBITT et al., 2016; PESSOA et al., 2016; KUMAR et al., 2017). Os cães braquicefálicos são os mais suscetíveis a essa condição e todos os dentes podem ser afetados, porém os mais acometidos são os primeiros pré-molares mandibulares e os caninos mandibulares e maxilares (NIEMIEC, 2008; HONZELKA et al., 2014; NIEMIEC, 2015; BABBITT et al., 2016).

Geralmente, o único sinal clínico é a ausência do dente afetado mas se o processo não for tratado podem ocorrer complicações como a formação de cistos odontogênicos, destruição óssea local, perda de dentes adjacentes, distorção da mandíbula ou maxila, e mesmo neoplasias (EDSTROM et al., 2013; BABBITT et al., 2016; THATCHER, 2017).

O tratamento consiste na exodontia do dente acometido, ou a realização de operculotomia, nos casos em que a única barreira impeditiva à erupção for a gengiva (EDSTROM et al., 2013; CARLE; SHOPE, 2014; DOMNICK, 2014; FULTON et al., 2014; NIEMIEC, 2015; KUMAR et al., 2017).

O cisto dentígero é o cisto odontogênico benigno mais comum em cães. Todavia, sua incidência é baixa, e está associado a dentes inclusos, ocorrendo principalmente no primeiro pré-molar mandibular de cães braquicefálicos e de pequeno porte (BELLEZZA et al., 2008; KUYAMA et al., 2009; D'ASTOUS, 2011; MACGEE et al., 2012; HONZELKA et al., 2014; MACGEE, 2014; VOELTERRATSON et al., 2015; REQUICHA et al., 2015; BABBITT et al., 2016; HOLLY et al., 2018; HOYER et al., 2016; PERRY, 2017).

Os cistos dentígeros podem alcançar tamanhos significativos durante sua lenta expansão, levando a fraturas patológicas decorrentes de destruição óssea, invasão da cavidade nasal, reabsorção ou problemas endodônticos em dentes vizinhos, e podem em alguns casos se tornar malignos (D`ASTOUS, 2011; MACGEE 
et al., 2012; MACGEE, 2014; MOORE; NIEMIEC, 2014; VOELTER-RATSON et al., 2015; NIEMIEC, 2015; BABBITT et al., 2016; PERRY, 2017; THEYSE, 2017).

Os animais acometidos geralmente não apresentam sinais clínicos além da hipodontia. No entanto, em lesões mais avançadas, pode ocorrer assimetria facial e presença de uma massa flutuante ou firme (D`ASTOUS, 2011; MACGEE et al., 2012).

Para o diagnóstico dos cistos dentígeros é essencial a realização de radiografias intra-orais, que permitem observar uma lesão circular radioluscente com um córtex bem definido. Além disso, recomenda-se realizar exame histolopatológico do material extraído (KUYAMA et al., 2009; D‘ ASTOUS, 2011; MACGEE et al., 2012; HONZELKA et al., 2014; MACGEE, 2014). Por outro lado, grandes cistos dentígeros podem ser confundidos com granulomas, abscessos, lesões fibro-ósseas, tumores e outros tipos de cistos (KUYAMA et al., 2009; D‘ASTOUS, 2011; HONZELKA et al., 2014).

O tratamento consiste na exodontia do dente incluso seguida de uma curetagem meticulosa. Porém, em caso de cistos grandes, é necessário realizar primeiro marsupialização e drenagem do mesmo (D‘ ASTOUS, 2011; HONZELKA et al., 2014; NIEMIEC, 2015; BABBITT et al., 2016).

O objetivo deste artigo é relatar um caso em que um cão apresentou persistência de três dentes decíduos e presença de dois dentes inclusos, sendo um deles associado a um cisto dentígero.

\section{RELATO DO CASO}

Foi atendido no Hospital Veterinário da Universidade Federal do Paraná (HVUFPR), em Curitiba - PR, um cão macho castrado da raça Shih-Tzu, com cinco anos de idade e pesando $7,4 \mathrm{~kg}$, com a queixa de halitose e presença de cálculo dental. Durante a consulta, a tutora afirmou que o animal nunca havia realizado procedimentos odontológicos e nem possuíam o hábito de realizar higienização oral no cão. A mesma também afirmou que o cão possuía bom estado de saúde, sem doenças anteriores e que vacinação e vermifugação encontravam-se atualizadas. $O$ cão se alimentava exclusivamente de ração seca, mas eventualmente recebia biscoitos.

Durante o exame físico geral não foi encontrada nenhuma anormalidade. No exame físico odontológico verificou-se ausência de assimetria da cabeça, aumento de volume, fístula, secreção nasal e gengivite. O animal apresentava oclusão prognata, presença do dente decíduo 503, halitose leve, com presença de cálculo dental e doença periodontal de Grau I, conforme a classificação do American Veterinary Dental College (AVDC). Língua, saliva, palato, lábios e mucosas não possuíam alterações. Foi indicado o tratamento periodontal e exodontia do dente decíduo em questão. Adicionalmente, foram requisitadas análises laboratoriais complementares, constituído por hemograma e exames bioquímicos, como alanina aminotransferase (ALT), fosfatase alcalina (FA), creatinina, ureia, proteínas totais (PT) e suas frações. Os resultados apresentaram-se dentro dos níveis de referência normal para a espécie.

Durante a consulta pré-anestésica, o anestesista novamente avaliou a animal e avaliou os exames, constatando que o mesmo encontrava apto para a cirurgia. $O$ procedimento foi realizado mediante utilização de anestesia inalatória com 
administração de isoflurano ${ }^{1}$ em vaporizador universal com circuito semi-fechado. Para medicação pré-anestésica administrou-se pela via intramuscular (IM) uma associação de acepromazina ${ }^{2}(0,025 \mathrm{mg} / \mathrm{kg})$ e metadona ${ }^{3}(0,2 \mathrm{mg} / \mathrm{kg})$. Realizou-se então cateterismo da veia cefálica direita, sendo a indução anestésica realizada com injeção intravenosa (IV) de propofol $^{4}(4 \mathrm{mg} / \mathrm{kg})$. A seguir foi realizada intubação orotraqueal e iniciou a administração de isoflurano.

Previamente aos procedimentos cirúrgicos realizou-se bloqueio anestésico infraorbitário e mandibular bilateralmente, empregando lidocaína sem vasoconstritor ${ }^{5}$ $(0,1 \mathrm{~mL} / \mathrm{kg})$. Durante toda a anestesia o paciente foi monitorizado em relação frequência cardíaca (FC), pressão arterial sistólica (PAS), frequência respiratória $(\mathrm{FR})$, saturação parcial de oxigênio $\left(\mathrm{SpO}_{2}\right)$ e temperatura retal, não ocorrendo nenhuma intercorrência.

A anestesia possibilitou confirmar a classificação de Grau I para cálculo dental e doença periodontal, além de verificar a presença dos dentes decíduos 503, 603 e 604 e ausência dos dentes 305 e 405 (Figuras 4 e 5). Em seguida, foi realizada limpeza dental do lado direito da arcada dentária, mediante raspagem com scaler ultrassônico. Depois disso, realizou-se avaliação individual de cada dente, empregando uma sonda milimetrada.

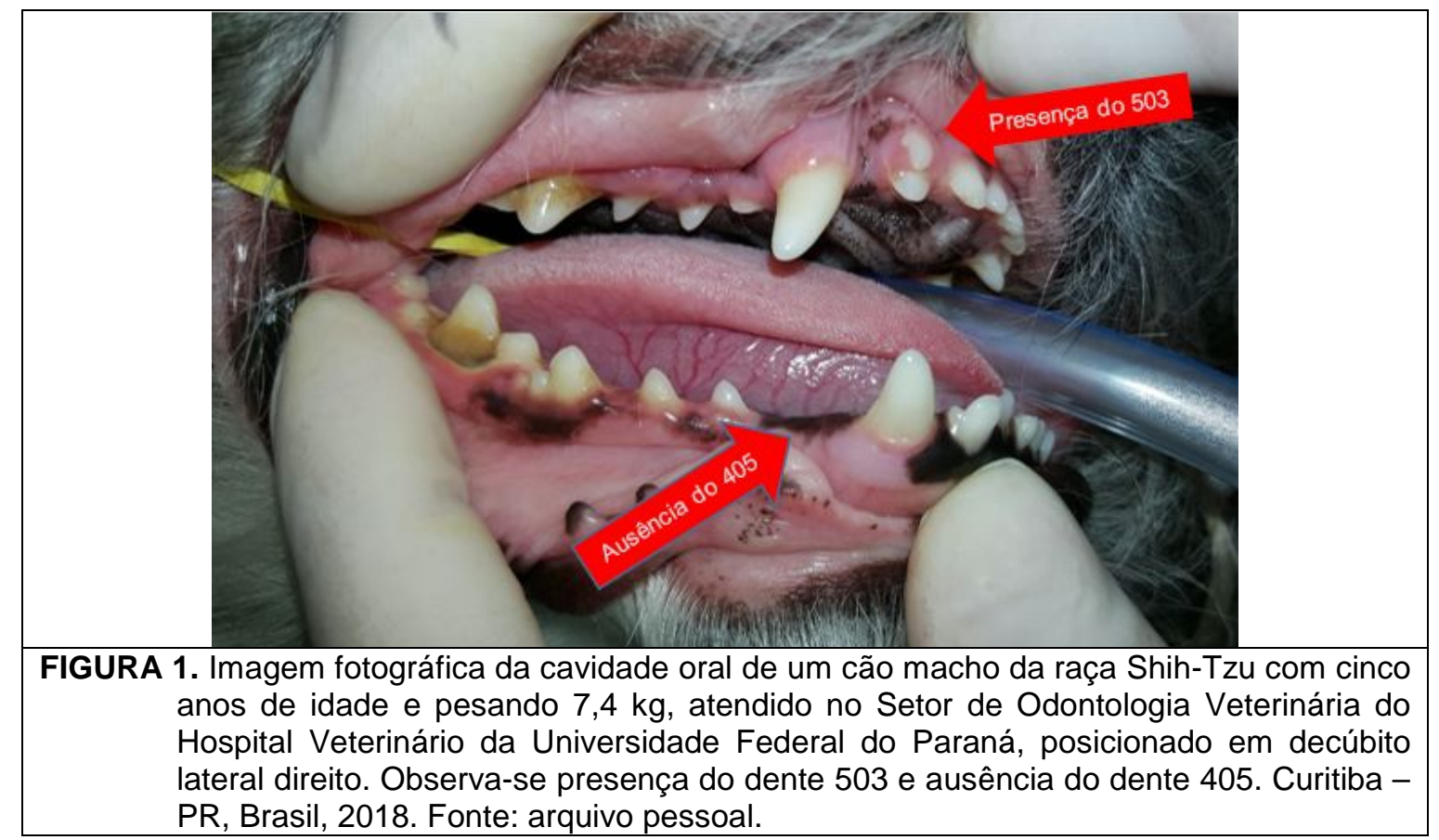

\footnotetext{
1 Isoflurano, Lab. BioChimico, Itatiaia - RJ.

${ }^{2}$ Acepromazina, Lab. Vetnil, Louveira - SP.

${ }^{3}$ Metadona, Lab Cristália, Itapira - SP.

${ }^{4}$ Propofol, Lab Cristália, Itapira - SP.

${ }^{5}$ Lidocaína sem vasoconstritor, Lab. Novafarma, Anápolis - GO.
} 


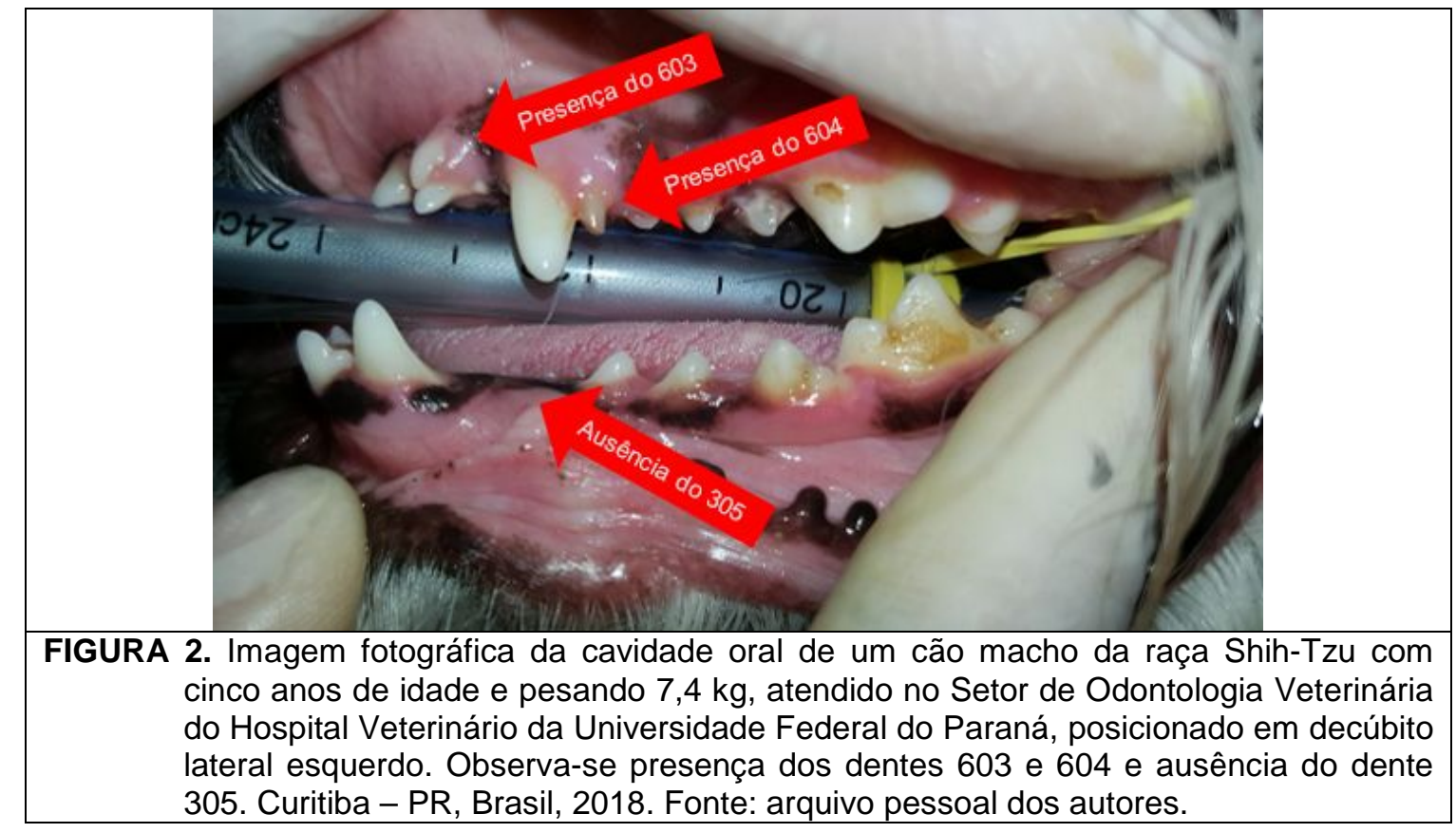

Posteriormente, foi radiografada a região dos dentes incisivos superiores (Figura 6), evidenciando a presença dos dentes decíduos 503, 603 e 604, e a região do primeiro pré-molar inferior esquerdo (Figuras 7 e 8), para investigar a causa da ausência do dente 405, constatando-se que o mesmo se encontrava incluso em posição horizontal, levemente reabsorvido. Foram então extraídos os dentes $503 \mathrm{e}$ 405 (Figuras 9 e 10), e a seguir foi realizado polimento com pasta profilática e mudança na posição do animal.

Todas as radiografias realizadas foram reveladas por meio de scanner específico para leitura desse tipo de imagem. Todas as áreas de exodontia, em ambos os lados, foram suturadas com fio absorvível de ácido poliglicólico ${ }^{6}$ número 40 , usando sutura simples interrompida. A Figura 16 apresenta os dentes extraídos.

\footnotetext{
${ }^{6}$ Fio de sutura, Bioline, Anápolis - GO.
} 

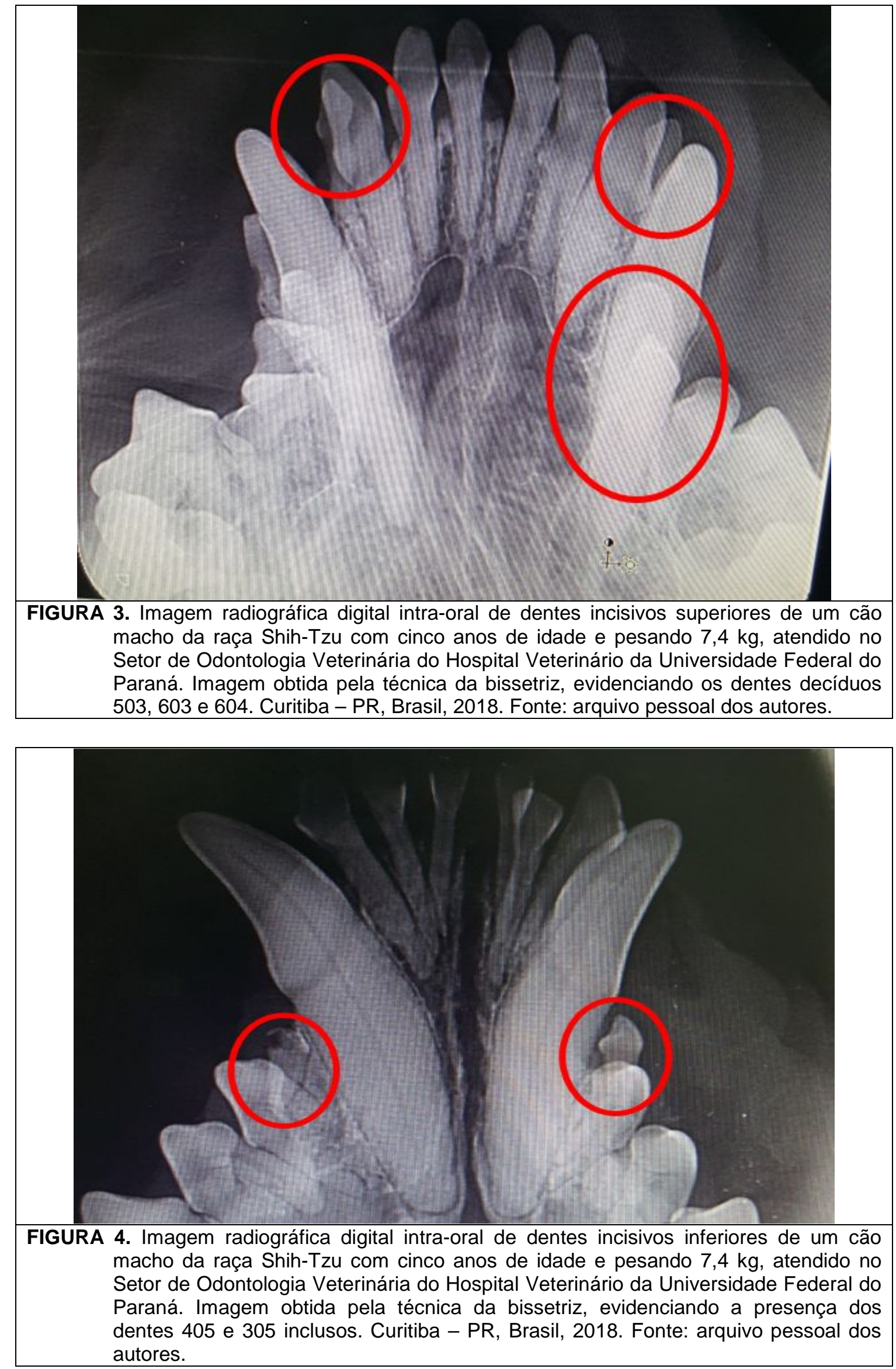

ENCICLOPÉDIA BIOSFERA, Centro Científico Conhecer - Goiânia, v.16 n.29; p. 1609 

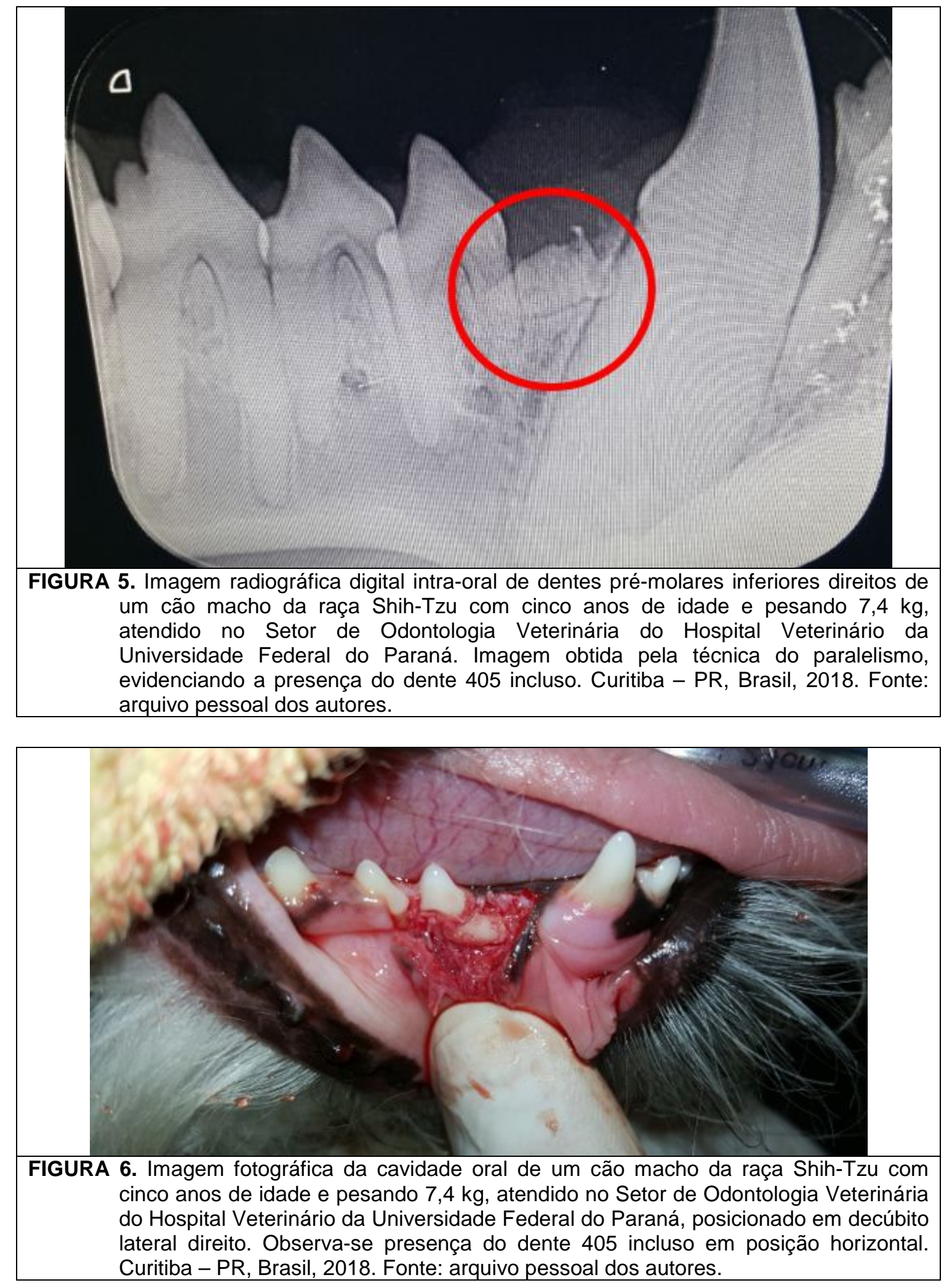


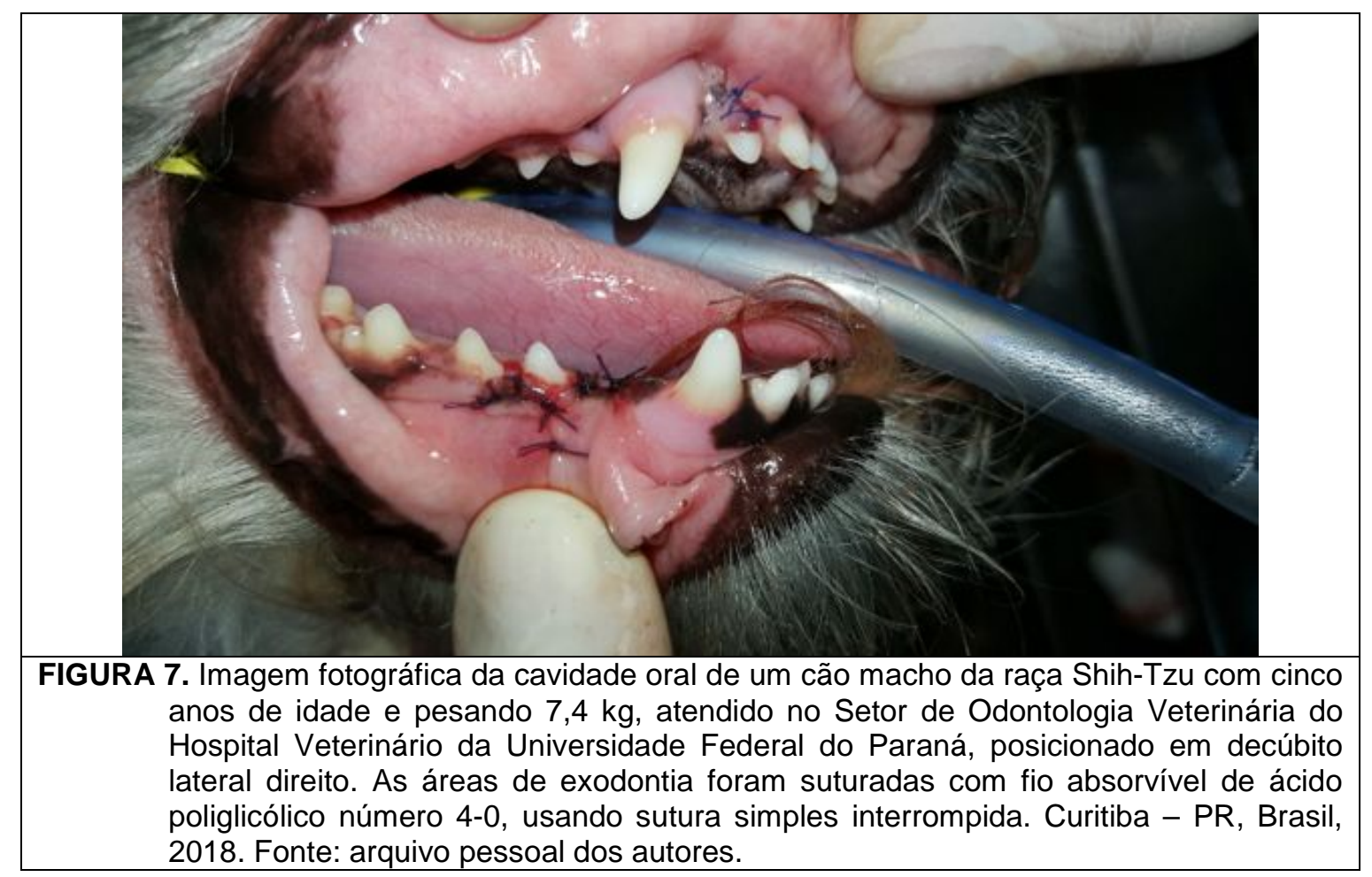

No lado esquerdo da arcada dentária, foi primeiramente realizada a limpeza dental mediante raspagem com scaler ultrassônico, seguida da avaliação individual dos dentes, empregando-se uma sonda milimetrada. Foi radiografada a região do primeiro pré-molar inferior esquerdo (305), para investigar a causa da ausência deste dente (Figura 11), e constatou-se que esse elemento dental encontrava-se incluso, em posição horizontal, com início de formação de um cisto dentígero. Foram então extraídos os dentes 603, 604 e 305 (Figuras 12, 13, 14 e 15), suturadas as áreas de exodontia, e finalmente realizado polimento com pasta profilática.

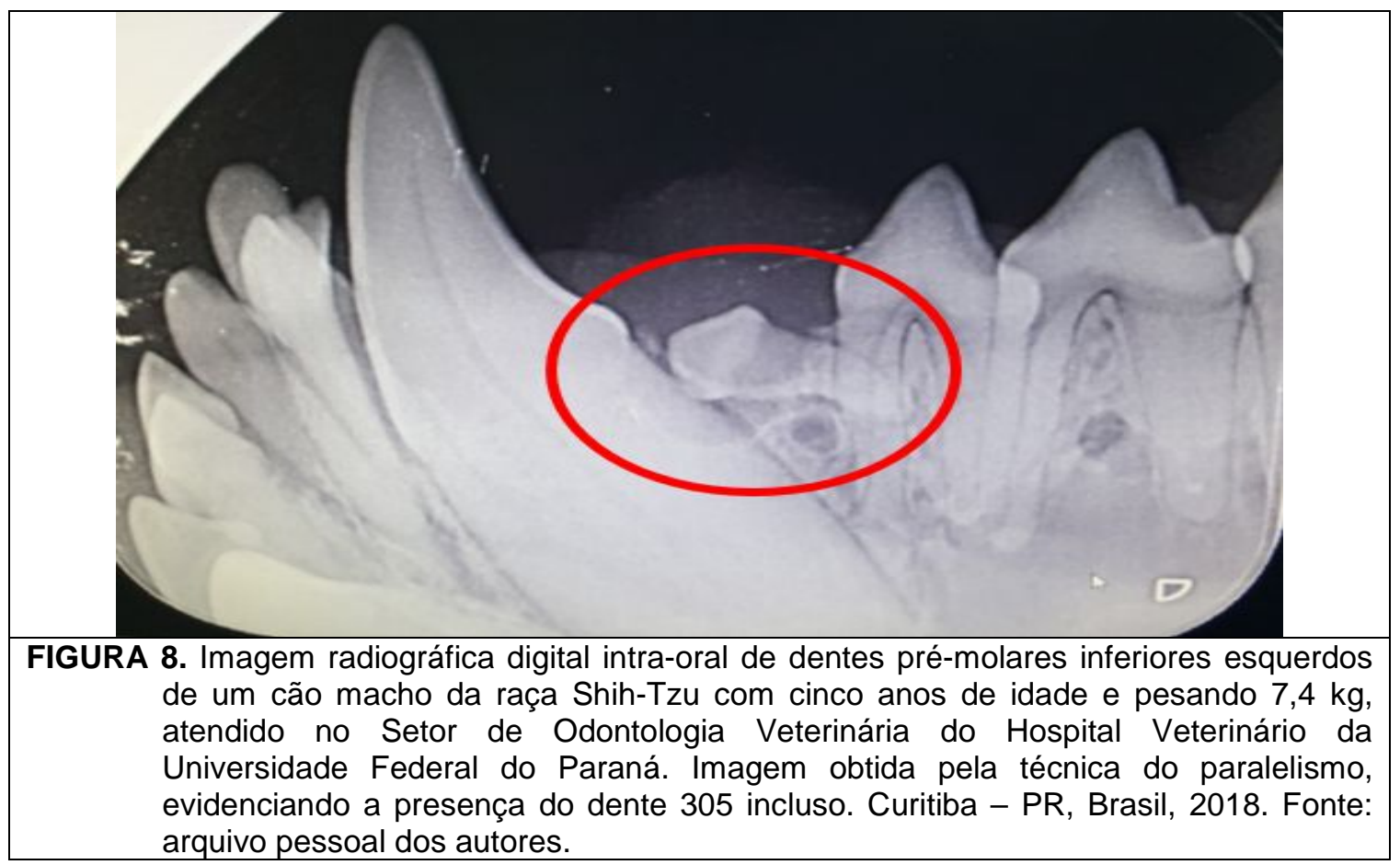

ENCICLOPÉDIA BIOSFERA, Centro Científico Conhecer - Goiânia, v.16 n.29; p. 1611 

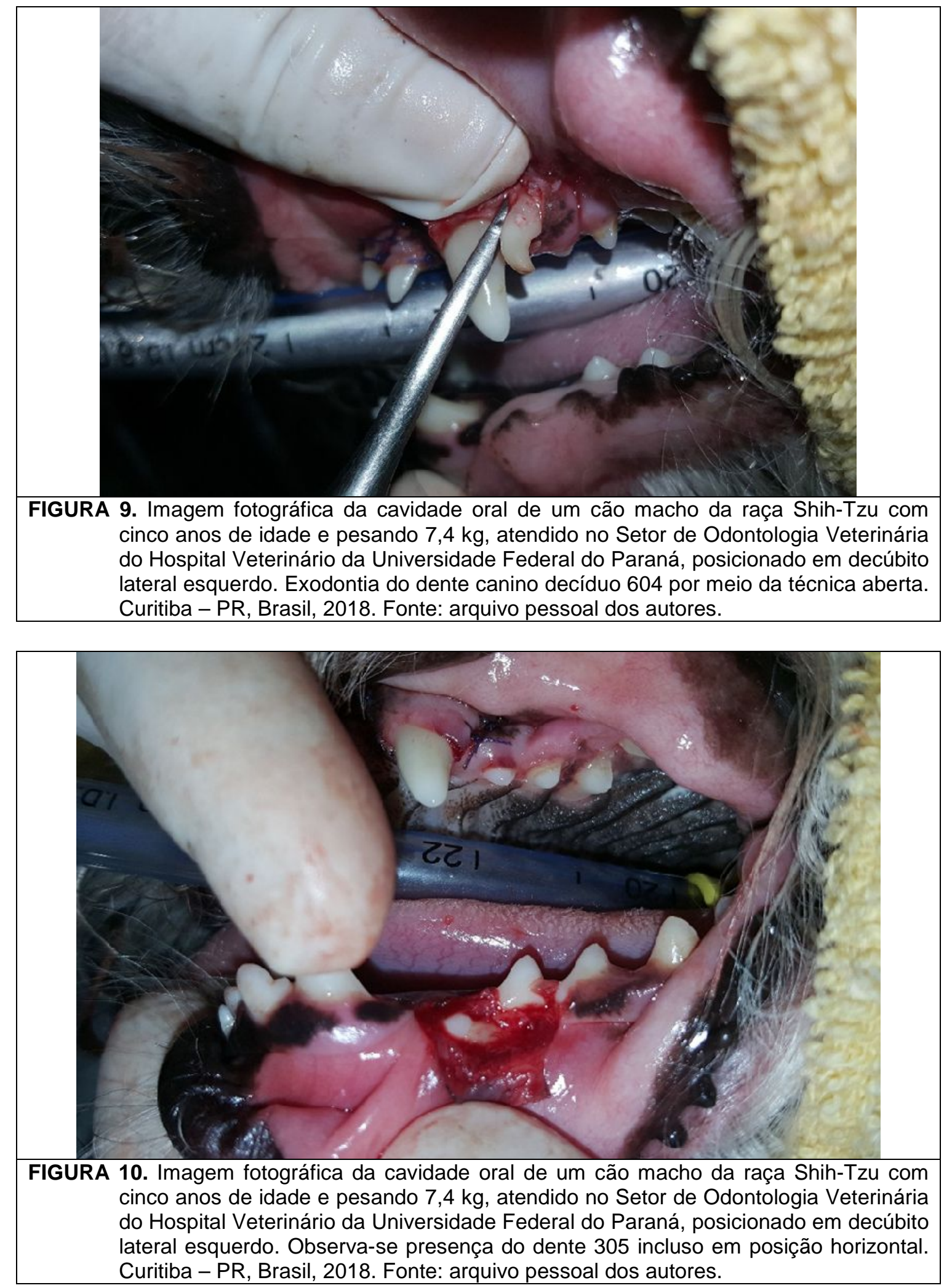


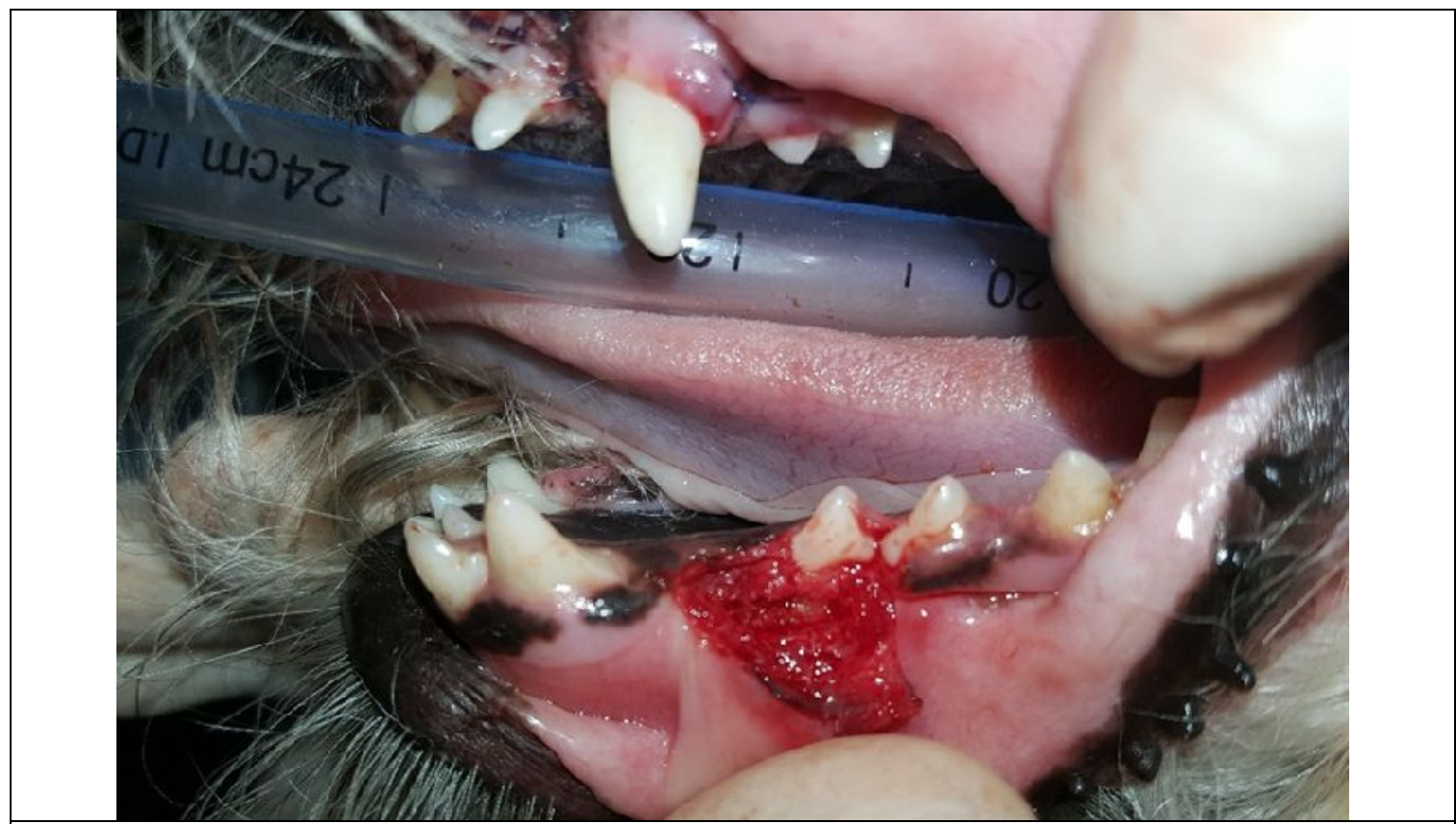

FIGURA 11. Imagem fotográfica da cavidade oral de um cão macho da raça Shih-Tzu com cinco anos de idade e pesando $7,4 \mathrm{~kg}$, atendido no Setor de Odontologia Veterinária do Hospital Veterinário da Universidade Federal do Paraná, posicionado em decúbito lateral esquerdo. Cavidade residual decorrente da exodontia do dente 305 incluso, onde observa-se início de alterações provenientes de formação cística. Curitiba PR, Brasil, 2018. Fonte: arquivo pessoal dos autores.

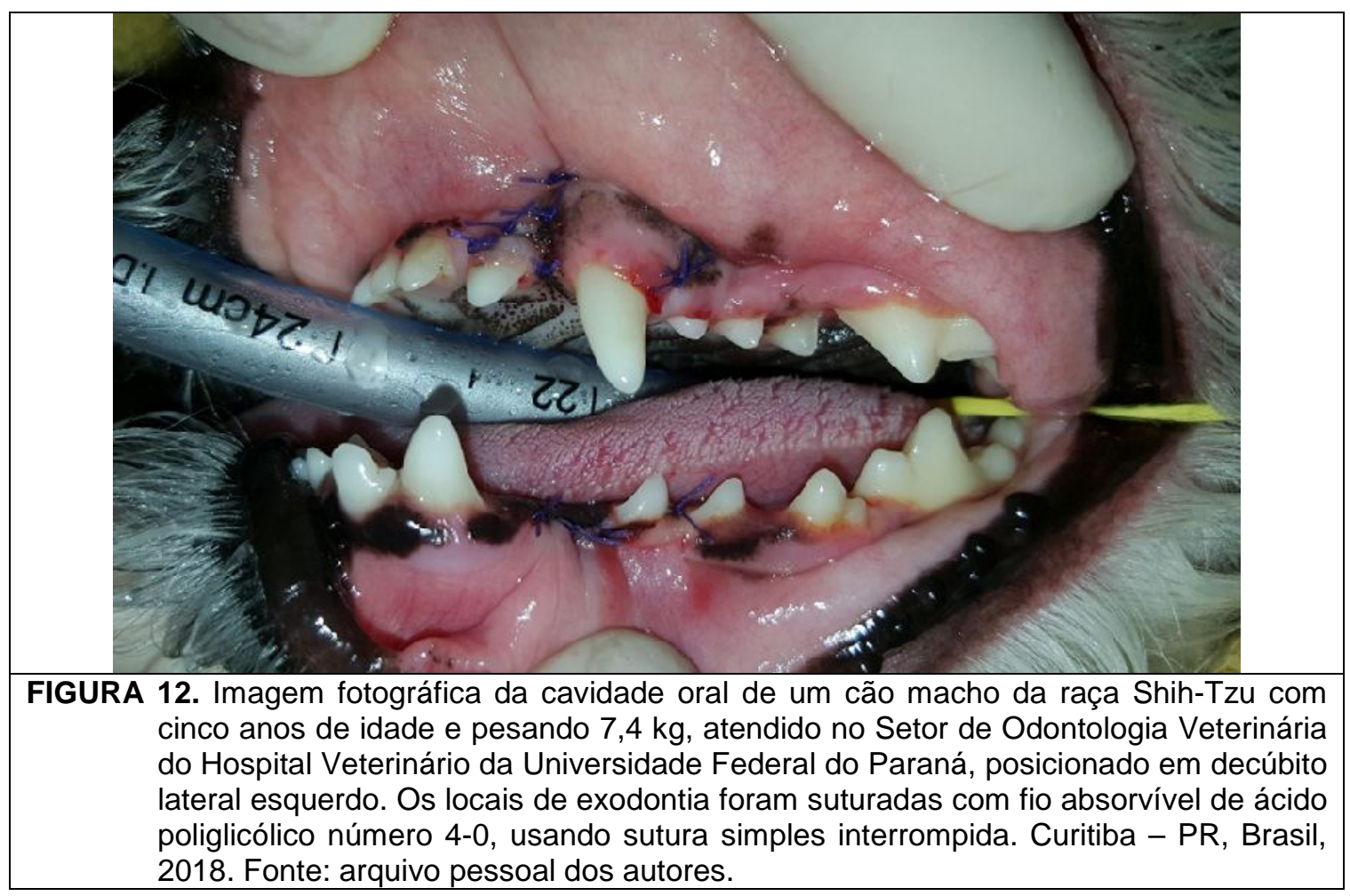




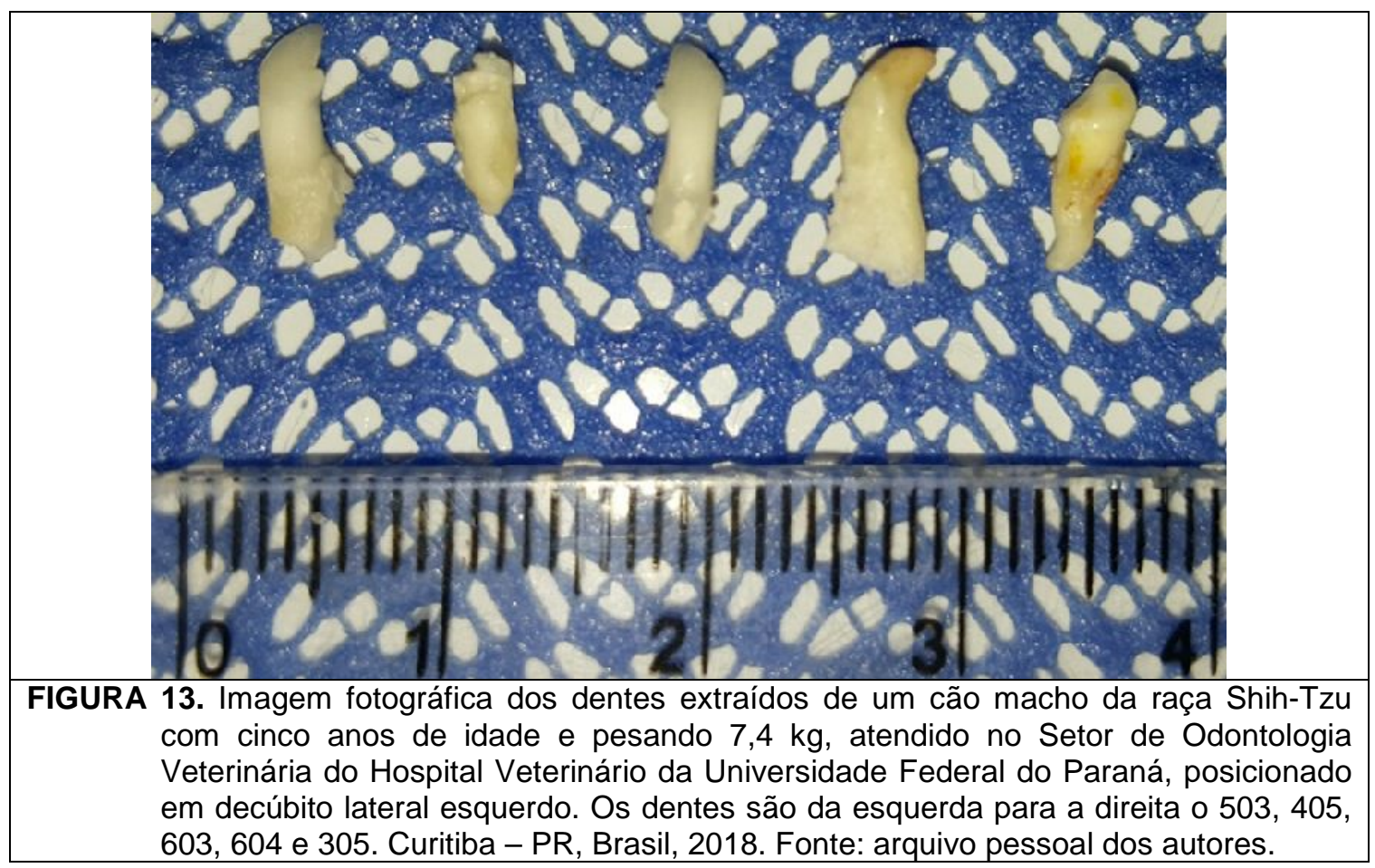

Como medicação pós-operatória prescreveu-se cloridrato de clindamicina ${ }^{7}$ (37,5 mg a cada 12 horas, durante sete dias, por via oral - PO), cloridrato de ranitidina $^{8}(15 \mathrm{mg}$ a cada 12 horas, durante sete dias, PO, 20 minutos antes da clindamicina), meloxicam ( $1,0 \mathrm{mg}$ a cada 24 horas, durante dois dias, $\mathrm{PO}$ ) e dipirona gotas (200 mg a cada 12 horas, durante dois dias, PO). Também foi indicado o uso tópico de solução oral de digluconato de clorexidina a $0,12 \%$ (duas vezes ao dia, durante cinco dias) e escovação diária com creme dental, para a limpeza de todas as superfícies dentais e gengivais, e alimentação pastosa durante cinco dias.

$\mathrm{Na}$ reavaliação clínica, ocorrida 13 dias após a intervenção, a tutora relatou que o animal manteve-se em boas condições gerais, mostrando apatia e apresentando emese e tosse nos primeiros dias. Alimentou-se normalmente com a dieta pastosa e recebeu todos os medicamentos prescritos corretamente, porém não permitiu o uso do antisséptico oral e a prática da escovação. Ao exame físico, os parâmetros vitais analisados encontravam-se normais e no exame oral observou-se total cicatrização nos locais de exodontia, presença de alguns pontos de sutura e ausência de cálculo dental, placa bacteriana e halitose.

Por fim, foi indicado o uso exclusivo de alimento industrializado seco, e a tutora foi orientada quanto à correta realização da higiene oral com creme dental apropriado para cães aplicado com gaze ou escova dental também apropriada para cães.

\section{RESULTADOS E DISCUSSÃO}

Os cães que mais apresentam persistência de dentes decíduos, presença de dentes inclusos e cistos dentígeros são os braquicefálicos de pequeno porte, como o paciente deste caso. Os sinais clínicos e lesões observados corroboram com as

\footnotetext{
${ }^{7}$ Oralguard®, Lab. CEPAV, São Paulo - SP.

${ }^{8}$ Antak®, Lab. Glaxo Smith Kline, Rio de Janeiro - RJ.
} 
descritas na literatura (NIEMIEC, 2008; KIM et al., 2013; FULTON et al., 2014; HONZELKA et al., 2014; SANTOS et al., 2014; NIEMIEC, 2015; BABBITT et al., 2016; THATCHER, 2017).

O primeiro pré-molar é um dos primeiros dentes permanentes a eclodir, por isso Babbitt et al. (2016) indicam uma maior atenção ao realizar o exame oral de braquicefálicos de pequeno porte, uma vez que estes são mais predispostos a ocorrência de dentes inclusos e cistos dentígeros. Essa predisposição ocorre devido as características anatômicas destes animais, que devido ao espaço insuficiente para a erupção de todos os dentes alguns acabam inclusos (KIM et al., 2013).

Foram realizadas radiografias dos dentes decíduos do paciente, que auxiliaram na extração dos mesmos, conforme proposto por Santos et al. (2014). Quando há ausência de dentes em um cão, é essencial a realização de radiografias para investigar a causa etiológica (LEITE et al., 2011; KIM et al., 2013; DOMNICK, 2014; FULTON et al., 2014; HONZELKA et al., 2014; NIEMIEC, 2015; BABBITT et al., 2016; PERRY, 2017). Neste contexto, foi radiografado a região dos primeiros pré-molares bilateralmente onde descobriu-se que o 305 e 405 encontravam-se inclusos. Além disso, percebeu-se uma área radioluscente associada a coroa do 305, sendo um sinal indicativo de cisto dentígero (D‘ASTOUS, 2011; HONZELKA et al., 2014; THATCHER, 2017).

Como recomendado por diversos autores, foi realizada a extração dos dentes decíduos e inclusos do animal, além de uma curetagem meticulosa do local de extração do 305, que possuía sinais radiográficos indicativos de cisto dentígero (NIEMIEC, 2008; KIM et al., 2013; DOMNICK, 2014; FULTON et al., 2014; HONZELKA et al., 2014; SANTOS et al., 2014; BABBITT et al., 2016; THATCHER, 2017).

Assim como indicado por Santos et al. (2014), foi realizado antibioticoterapia e analgesia, além de solicitar limpeza com solução oral de clorexidina durante a cicatrização. Entretanto, segundo a tutora, a limpeza não pode ser realizada já que o animal não permitiu o procedimento.

No retorno, foi relatado que o animal apresentou êmese e tosse nos primeiros dias após o procedimento odontológico. Segundo Nelson e Couto (2015), quase todos os fármacos podem causar vômito, mas entre os principais encontram-se os antiinflamatórios não esteroidais (AINEs). O meloxicam é um AINE e foi prescrito por dois dias para o paciente, sendo a provável causa da êmese. Já a tosse ocorreu em decorrência da intubação orotraqueal (CAMPOS et al., 2016).

\section{CONCLUSÃO}

Neste caso, as únicas queixas durante a consulta foram a presença de halitose e cálculo dental. Entretanto, ao realizar o exame oral foi possível identificar outros problemas como, oclusão prognata e presença do dente decíduo 503. Foi indicado o tratamento periodontal e exodontia do dente decíduo em questão. Com a realização de um novo exame oral sob anestesia foi possível identificar mais dois dentes decíduos (603 e 604) e a ausência do 305 e 405. Após a realização de radiografias, verificou-se que esses primeiros pré-molares estavam inclusos, sendo um deles com início de formação cística. Todos os dentes decíduos e inclusos foram extraídos com êxito e no retorno observou-se total cicatrização nos locais de exodontia e ausência de cálculo dental, placa bacteriana e halitose. 


\section{REFERÊNCIAS}

AVDC - American Veterinary Dental College. Stages of pet periodontal disease. Disponível em: <http://avdc.org/AFD/five-stages-of-pet-periodontaldisease/>. Acesso em: 14 dez/ 2018.

BABBITT, S.G.; VOLKER, M.K.; LUSKIN, I.R. Incidence of radiographic cystic lesions associated with unerupted teeth in dogs. Journal of veterinary dentistry, Thousand Oaks, v. 33, n. 4, p. 226-233, 2016.

BELLEZZA, E.; ANGELI, G.; LEONARDI, L.; RASTRELLI, O.; DI MARI, W. et al. A case of a mandibular dentigerous cyst in a German Shepherd dog. Veterinary research communications, Dordrecht, v. 32, n. 1, p. 235-237, 2008.

CAMPOS, N.F.; BOUGO, G.C.; GAMA, A.C.C.; VICENTE, L.C.C. Efeitos da intubação orotraqueal na voz e deglutição de adultos e idosos. Distúrbios da Comunicação, São Paulo, v. 28, n. 4, p. 597-608, dez/ 2016.

CARLE, D.; SHOPE, B. Soft tissue tooth impaction in a dog. Journal of veterinary dentistry, Thousand Oaks, v. 31, n. 2, p. 96-105, 2014.

D'ASTOUS, J. An overview of dentigerous cysts in dogs and cats. Canadian Veterinary Medical Association, Ottawa, v. 52, p. 905-907, ago/ 2011.

DOMNICK, E.D. Diagnostic imaging in veterinary dental practice. Journal of the American Veterinary Medical Association, Schaumburg, v. 245, n. 3, p. 281-283, ago/ 2014

EDSTROM, E.J.; SMITH, M.M.; TANEY, K. Extraction of the impacted mandibular canine tooth in the dog. Journal of veterinary dentistry, Thousand Oaks, v. 30, n. 1, p. 56-61, 2013.

FULTON, A.J.; FIANI, N.; VERSTRAETE, F.M. Canine pediatric dentistry. The veterinary clinics of North America. Small animal practice, Maryland Heights, v. 44, p. 303-324, mar/ 2014.

HOLLY, V.L.; ZWICKER, L.A.; STARRAK, G.; LEIS, M.L.; BAUER, B.S. et al. Odontogenic parakeratinized cyst resulting in exophthalmos and palatine, maxillary, and zygomatic bone erosion in a dog. Veterinary ophthalmology, Oxford, v. 21, n. 5, p. 539-543, set/ 2018.

HONZELKA, S.R.; KRESSIN, D.J.; CHAMBERLAIN, T.P. Modified conservative treatment of an extensive dentigerous cyst in a dog. Journal of veterinary dentistry, Thousand Oaks, v. 31, n. 4, p. 249-254, 2014.

HOYER, N.K.; BANNON, K.M.; BELL, C.M.; SOUKUP, L.W. Extensive maxillary odontomas in two dogs: diagnosis, pathology, and management. Journal of veterinary dentistry, Robbinsville, v. 33, n. 4, p. 234-242, 2016. 
KIM, C.G.; LEE, S.Y.; KIM, J.W.; PARK, H.M. Assessment of dental abnormalities by full-mouth radiography in small breed dogs. Journal of the American Animal Hospital Association, South Bend, v. 49, n. 1, p. 23-30, jan/fev 2013.

KRESSIN, D. Oral examination of cats and dogs. Compendium on continuing education for the practicing veterinarian, Yardley, v. 31, n. 2, p. 72-85, fev/ 2009.

KUMAR, V.; LALZAWMLIANA, V.; TRIPATHY, B.; JHA, R.K.; MUKHERJEE, P. et al. Surgical management of impacted premolar and molar teeth in a german shepherd dog. Indian journal of animal health, Calcutá, v. 56, n. 1, p. 101-104, 2017.

KUYAMA, K.; HAYASHI, K.; FUFITA, S.F.; SATOH, I.; YAMAMOTO, H. Immunohistochemical Analysis of a dentigerous cyst in a dog. Journal of veterinary dentistry, Thousand Oaks, v. 26, n. 2, p. 106-109, 2009.

LEITE, C.A. L.; ASSIS, L.S.; ARAUJO, I.M.G.; SAMPAIO, G.R.; GUIMARÃES, P.T.C. et al. Técnicas radiográficas intra e extrabucal na avaliação dentária de cães com doença periodontal. Arquivo brasileiro de medicina veterinária e zootecnia, Belo Horizonte, v. 63, n. 5, p. 1099-1103, 2011.

MACGEE, S. Endodontic therapy of a mandibular canine tooth with irreversible pulpitis secondary to dentigerous cyst. Journal of veterinary dentistry, Thousand Oaks, v. 31, n. 1, p. 30-39, 2014

MACGEE, S.; PINSON, D.M.; SHAIKEN, L. Bilateral dentigerous cysts in a dog. Journal of veterinary dentistry, Thousand Oaks, v. 29, n. 4, p. 242-249, 2012.

MOORE, J.I.; NIEMIEC, B. Evaluation of extraction sites for evidence of retained tooth roots and periapical pathology. Journal of the American Animal Hospital Association, Lakewood, v. 50, n. 2, p. 77-82, mar/abr 2014.

NELSON, R. W.; COUTO, C. G. Medicina interna de pequenos animais. 5. ed. Rio de Janeiro: Elsevier, 2015.

NIEMIEC, B.A. Oral pathology. Topics in companion animal medicine, New York, v. 23 , n. 2, p. 59-71, mai/ 2008.

NIEMIEC, B.N. Dental radiology series the importance of dental radiography. Today's Veterinary Practice, Gainesville, v. 5, n. 3, p. 18-25, mai/jun 2015.

PERRY, A.J. Dental radiology in dogs and cats. Companion animal, London, v. 22, n. 3, p. 150-159, mar/ 2017.

PESSOA, L.M. B.; ROZA, M.; FARIAS, A.; JESUS, P.H.; CAMPBELL, R.C. et al. Extraction of unerupted maxillary canine teeth in a maned wolf (Chrysocyon brachyurus). Case reports in veterinary medicine, London, v. 2016, p. 1-3, jul 2016. 
REQUICHA, J.F.; PIRES, M.A.; ALBUQUERQUE, C.M.; VIEGAS, C.A. Neoplasias da cavidade oral do cão - Breve revisão. Revista brasileira de medicina veterinária, Rio de Janeiro, v. 37, n. 1, p. 41-46, jan/mar 2015.

SANTOS, I.F.C.; BENE, M.; GASPAR, B.; BAMBO, O.; CARDOSO, J.M.M. Persistência de dentes decíduos em cão (Canis familiaris): relato de caso. Revista científica da Universidade Eduardo Mondiane, Série: Ciências agronômicas, florestais e veterinárias, Maringá, v. 1, n. 1, p. 75-81, 2014.

THATCHER, G. Oral Surgery: Treatment of a dentigerous cyst in a dog. Canadian Veterinary Medical Association, Ottawa, v. 58, p. 195-199, fev/ 2017.

THEYSE, L.F.H. Management of oral tumours. The thai journal of veterinary medicine, Bangkok, v. 47, p. 81, 2017.

VOELTER-RATSON, K.; HAGEN, R.; GRUNDMANN, S.; SPIESS, B.M. Dacryocystitis following a nasolacrimal duct obstruction caused by an ectopic intranasal tooth in a dog. Veterinary ophtalmology, Oxford, v. 18, n. 5, p. 433-436, 2015. 